\title{
Nature of magnetism in thiol-capped gold nanoparticles investigated with Muon spin rotation
}

Cite as: Appl. Phys. Lett. 112, 053105 (2018); https://doi.org/10.1063/1.5017768

Submitted: 30 November 2017 . Accepted: 16 January 2018 . Published Online: 31 January 2018

M. H. Dehn, D. J. Arseneau (D, T. Buck, D. L. Cortie, D. G. Fleming, S. R. King, W. A. MacFarlane, A. M. McDonagh, R. M. L. McFadden (D, D. R. G. Mitchell, and R. F. Kiefl
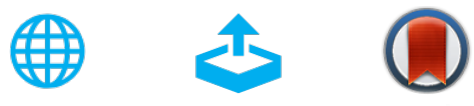

View Online

Export Citation

CrossMark

\section{ARTICLES YOU MAY BE INTERESTED IN}

Communication: Chemisorption of muonium on gold nanoparticles: A sensitive new probe of surface magnetism and reactivity

The Journal of Chemical Physics 145, 181102 (2016); https://doi.org/10.1063/1.4967460

Electronic and magneto-transport in chirality sorted carbon nanotube films

Applied Physics Letters 112, 053104 (2018); https://doi.org/10.1063/1.5011090

Detection of ultra-low oxygen concentration based on the fluorescence blinking dynamics of single molecules

Applied Physics Letters 112, 053101 (2018); https://doi.org/10.1063/1.5005157

\section{Lake Shore}

CRYOTRONICS

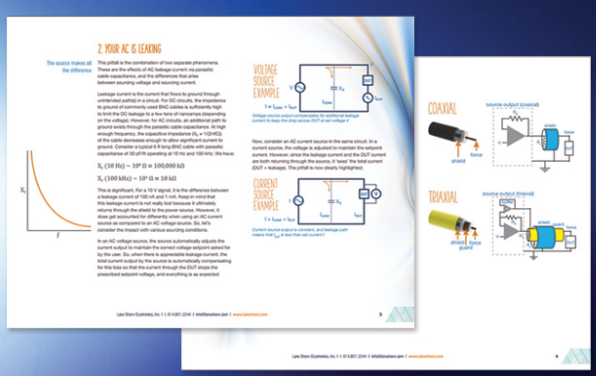

5 Electronic

- Measurement Pitfalls to Avoid

Get the whitepaper $\bullet$ 


\title{
Nature of magnetism in thiol-capped gold nanoparticles investigated with Muon spin rotation
}

\author{
M. H. Dehn, ${ }^{1, \text { a) }}$ D. J. Arseneau, ${ }^{2}$ T. Buck, ${ }^{3}$ D. L. Cortie, ${ }^{3, b)}$ D. G. Fleming, ${ }^{2,4}$ S. R. King, ${ }^{5}$ \\ W. A. MacFarlane, ${ }^{2,4}$ A. M. McDonagh, ${ }^{5}$ R. M. L. McFadden, ${ }^{4}$ D. R. G. Mitchell, ${ }^{6}$ \\ and R. F. Kiefl ${ }^{2,3, c)}$ \\ ${ }^{1}$ Physik-Department, Technische Universität München, James-Franck-Strasse 1, \\ 85748 Garching bei München, Germany \\ ${ }^{2}$ Triumf, 4004 Wesbrook Mall, Vancouver, British Columbia V6T 2A3, Canada \\ ${ }^{3}$ Department of Physics and Astronomy, University of British Columbia, Vancouver, \\ British Columbia V6T 1Z1, Canada \\ ${ }^{4}$ Department of Chemistry, University of British Columbia, Vancouver, British Columbia V6T 1Z1, Canada \\ ${ }^{5}$ School of Mathematical and Physical Sciences, University of Technology Sydney, Broadway, NSW 2007, \\ Australia \\ ${ }^{6}$ Electron Microscopy Centre, Australian Institute for Innovative Materials, University of Wollongong, \\ North Wollongong, NSW 2500, Australia
}

(Received 30 November 2017; accepted 16 January 2018; published online 31 January 2018)

\begin{abstract}
Muon spin rotation/relaxation measurements show clear evidence for magnetism in $2.2 \mathrm{~nm}$ gold nanoparticles capped with butanethiol. At low temperatures $(1.8 \mathrm{~K})$, there is significant spin relaxation which decreases as a function of both the applied longitudinal magnetic field and increasing temperature. The results indicate that there are spatially inhomogeneous electronic moments that fluctuate with a wide distribution of correlation times. Possible explanations are discussed. Published by AIP Publishing. https://doi.org/10.1063/1.5017768
\end{abstract}

Although there is uncertainty in both experiments and theory regarding the fundamental properties of gold nanoparticles (GNPs), they are nevertheless employed in many different areas including medicine, bio- and nanotechnology, and catalysis. ${ }^{1-3}$ A better understanding of their most elusive property, its magnetism, could open up new fields of application and contribute to the understanding of magnetism at a more fundamental level. Therefore, the nature and origin of magnetism in GNPs continue to be the subject of intense study and debate. ${ }^{4}$ Bulk gold exhibits only temperature independent diamagnetism $\left(-0.142 \times 10^{-6} \mathrm{emu} / \mathrm{g}\right)$ from conduction electrons which is much larger than the Pauli paramagnetism. ${ }^{5}$ In GNPs, a different magnetic behaviour is anticipated, as the Fermi wavelength is comparable to the particle size, and the energy level spacing between electronic levels is comparable to, or larger than, $k_{B T}$. On general grounds, one would expect paramagnetism from a system of weakly coupled NPs since those nanoparticles with an odd number of gold atoms must have at least one Bohr magneton. ${ }^{6-8}$ However, there is no consensus on either the experimental or the theoretical side, ${ }^{4}$ which may be attributed to the variable role of the capping material often used to prevent aggregation of GNPs. Examples include the study by Garitaonandia et al. who reported permanent ferromagnetism in thiol-capped GNPs, proposing a collective orientation of the spins of localized d-electrons through exchange coupling. ${ }^{9}$ Crespo et al. also reported ferromagnetism in thiol-

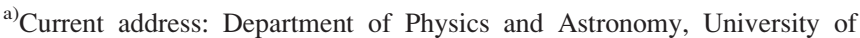
British Columbia, Vancouver, British Columbia V6T 1Z1, Canada. Electronic mail: mdehn@triumf.ca.

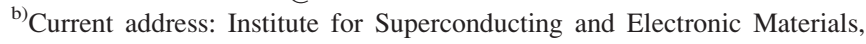
AIIM, University of Wollongong, Northfields Ave, Wollongong NSW 2522, Australia.

c)kiefl@triumf.ca
}

capped GNPs, whereas diamagnetic behaviour was seen in similar GNPs coated with a weakly interacting surfactant. The ferromagnetism was attributed to localized d-holes at the surface due to charge transfer to the strongly interacting thiol ligand. ${ }^{10}$ In contrast, Yamamoto et al. observed superparamagnetic behaviour in GNPs protected with a weakly interacting PAAHC polymer and reported a sizable reduction in the magnetization when changing the capping material to thiol. ${ }^{11}$ An additional intriguing feature of GNPs reported on by a majority of experimental studies is a puzzling temperature independency of the reported magnetism. ${ }^{4}$ These experimental results have led to new and interesting theoretical ideas. Hernando et al., for instance, have suggested that charge carriers introduced by the surface coating are confined to two dimensions, which results in a large collective moment and giant paramagnetism. ${ }^{12}$

This paper reports on a Muon Spin Rotation/Relaxation ( $\mu \mathrm{SR})$ study on butanethiol-capped GNPs $2.2 \mathrm{~nm}$ in diameter. Spin polarized positive muons are well known as a sensitive local probe of magnetism, ${ }^{13}$ including metal nanoparticles. ${ }^{14}$ Here, we find significant muon spin relaxation attributed to localized magnetic moments of electronic origin which are dilute and fluctuate with a very broad range of correlation times at $1.8 \mathrm{~K}$. The observed spin relaxation is described by a stretched exponential form with an average rate that decreases with increasing temperature. Our results indicate the presence of spatially non-uniformly distributed magnetic moments in thiol-capped GNPs.

Butanethiol-capped GNPs were synthesized at the University of Technology, Sydney, following a procedure by $\mathrm{Wu}$ et al., ${ }^{15}$ with a slight modification. This method was selected because it is capable of manufacturing relatively large quantities of small nanoparticles with a tight size distribution. Instead of vacuum drying and redissolving the particles in 
$20 \mathrm{ml}$ of cyclohexane, the centrifuged particles were washed with $50 \mathrm{ml}$ of methanol and centrifuged again and then placed in a vial to dry under a stream of nitrogen gas. This process yielded a total sample weight of $1.06 \mathrm{~g}$. Based on a thermogravimetric analysis, the thiol is $11 \%$ of the sample by mass.

High resolution scanning transmission electron microscopy (STEM) images were taken at the Australian Institute for Innovative Materials to measure the nanoparticle diameter and size distribution. For that study, specimens were prepared by micro-pipetting $80 \mu \mathrm{l}$ of a suspension of nanoparticles in toluene onto continuous carbon support films (on a copper grid). Specimens were air dried and transferred immediately to the microscope. Examination was carried out using a JEOL ARM200F aberration (probe) corrected instrument operating at $200 \mathrm{kV}$. A probe size of approximately $0.08 \mathrm{~nm}$ and a current of $30 \mathrm{pA}$ were used. A scanning transmission electron microscopy mode was used with high angle annular dark field (HAADF) and bright field (BF) signals being used. The HAADF signal enabled the direct imaging of atomic positions with an approximate intensity dependence on $Z^{2}$, where $Z$ is the atomic number. This provided high contrast between the gold nanoparticles and the carbon support film [Fig. 1(a)]. ${ }^{16}$ The average of 417 particles measured gives a mean radius $r=1.10 \pm 0.51 \mathrm{~nm}$. Figure 1(b) shows the distribution of radii.

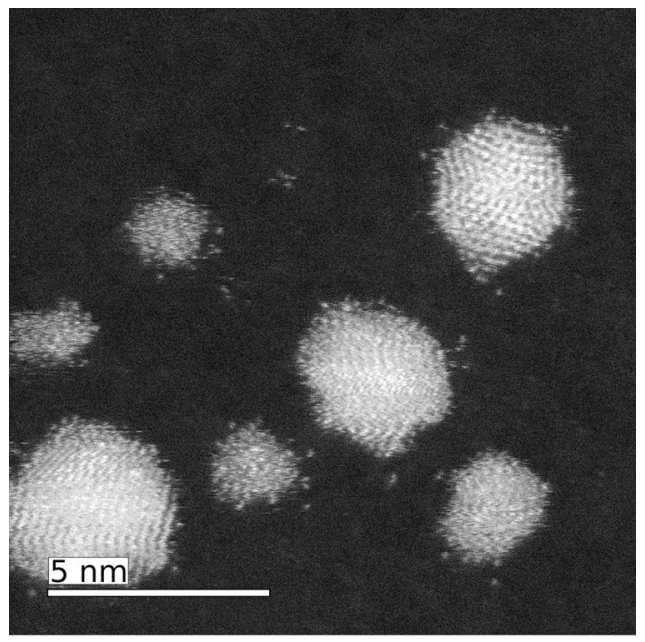

(a)

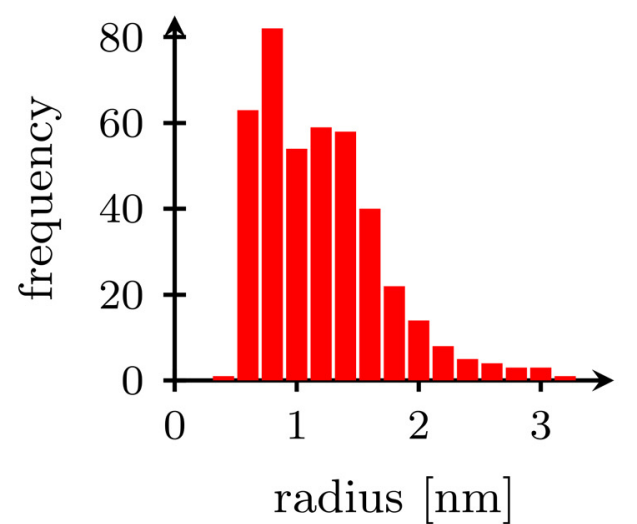

(b)

FIG. 1. (a) STEM HAADF image of the GNPs on a carbon support film and (b) size distribution based on 417 particles measured. The mean radius is $r=1.10 \pm 0.51 \mathrm{~nm}$.
A detailed description of the $\mu \mathrm{SR}$ technique can be found elsewhere. ${ }^{13}$ To summarize, highly spin polarized muons $\left(\mu^{+}\right)$ are implanted into the sample. The time evolution of the spin polarization is monitored by detecting the muon decay positrons, which are emitted preferentially along the muon spin direction at the time of decay. In a static magnetic field, the muon spin processes about the field direction at the Larmor frequency $\omega_{\mu}=\gamma_{\mu} B$, where $\gamma_{\mu}=2 \pi \cdot 0.01355 \mathrm{MHz} / \mathrm{G}$ is the muon gyromagnetic ratio. In general, the muon spin evolves in the total magnetic field, which is the sum of the applied field and any local internal magnetic field at the muon stopping site. The latter may also have a dynamic component, leading to spin relaxation. Thus, the observed $\mu \mathrm{SR}$ signal $S(t)$ contains information on both the quasi-static (static on the scale of the muon lifetime of $2.2 \mu \mathrm{s}$ ) and dynamic properties of the internal magnetic field in the sample.

The $\mu \mathrm{SR}$ experiments were carried out at TRIUMF's M20 beamline with the LAMPF spectrometer. The powder sample was mounted on a sample rod contained with thin Xray Mylar and inserted into a He gas-flow cryostat. Small auxiliary coils were used to zero the magnetic field to within $50 \mathrm{mG}$ prior to the experiment. In experiments employing external magnetic fields, one differentiates between the transverse field (TF) geometry, where the applied field is perpendicular to the initial muon spin polarization, and the longitudinal field (LF) configuration, where the applied field coincides with the initial polarization direction. Temperature scans were made in $B=23 \mathrm{G}$ TF and in $B=2 \mathrm{G}$ and $10 \mathrm{G}$ LFs. These were acquired in a spin rotated mode, i.e., with the initial muon spin polarization close to the vertical direction $(\hat{x})$ and perpendicular to the beam direction $(\hat{z})$. In addition, a LF scan up to $2 \mathrm{kG}$ was made at the base temperature of $1.78 \mathrm{~K}$ in a non-spin rotated mode with the initial polarization close to the beam direction $(\hat{z})$.

Figure 2(a) shows the muon spin precession signal in $B=23 \mathrm{G}$ TF. Note that the damping increases as the temperature is lowered. The observed signal cannot be described by a single exponential damping rate, indicating that there is a broad range of internal magnetic fields at the muon stopping site, consistent with a spatially inhomogeneous distribution of magnetic moments. The signal was fit assuming a stretched exponential relaxation envelope

$$
S^{\mathrm{TF}}(t)=A \exp \left[-\left(\lambda^{\mathrm{TF}} t\right)^{\beta}\right] \cos \left[\gamma_{\mu} B t+\phi\right],
$$

where $A$ is the amplitude, $\lambda^{\mathrm{TF}}$ the TF relaxation rate, $\beta$ the stretching parameter, and $\phi$ the initial phase. The fit results are shown in Figs. 2(b) and 2(c). Note that the relaxation rate $\lambda$ rises rapidly with decreasing temperature, whereas $\beta$ has a weaker temperature dependence. The small $\beta$ value at low temperature indicates a broad distribution of relaxation rates and thus a very inhomogeneous magnetic environment from the muon's perspective. Note that even a uniform orientation of moments located on the surface would give rise to a field distribution for the muon due to its variable stopping depths from the center of the sphere. For spherical GNPs, we expect that the stopping distribution is peaked close to the surface $\left(r^{2}\right.$-dependence), with a small constant tail into the thiol ligands. There is also some loss of initial amplitude at low 

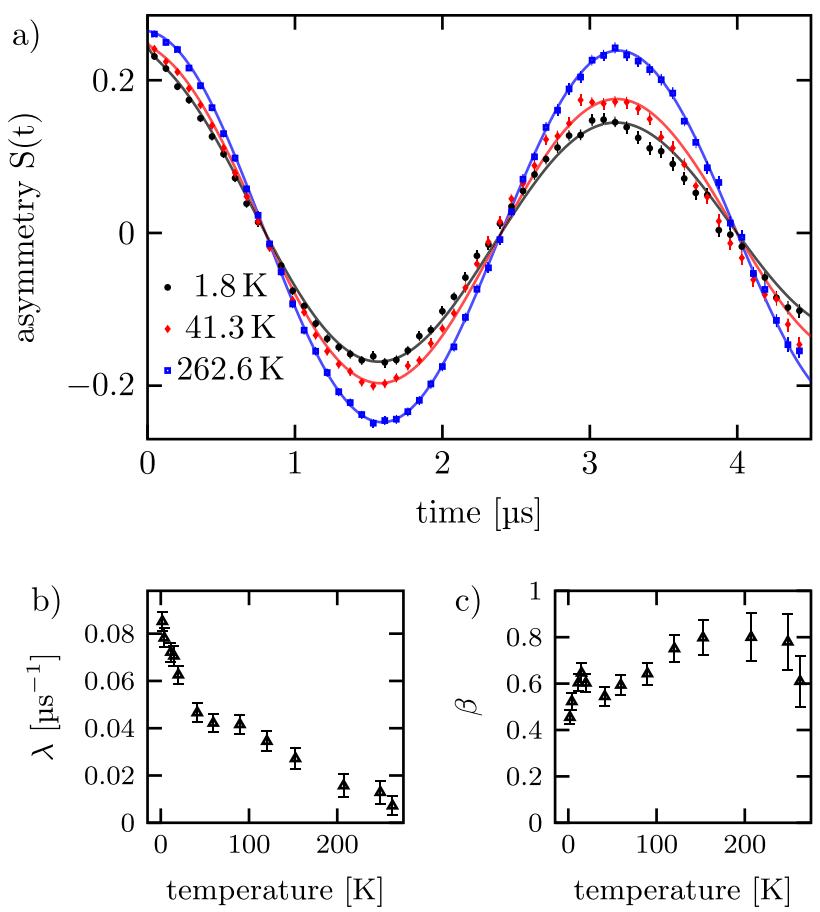

FIG. 2. (a) Asymmetry spectra in $B=23 \mathrm{G}$ TF at three temperatures. The colored lines are fits to a stretched exponential relaxation envelope [Eq. (1)]. (b) Relaxation rate $\lambda$ and (c) stretching parameter $\beta$ as obtained from this fit. With decreasing temperature, the relaxation rate increases and $\beta$ decreases, indicating the development of a highly inhomogeneous fluctuating internal field distribution.

temperature [Fig. 2(a)], which indicates that a small fraction of muons experiences very large internal fields.

Spectra were also obtained as a function of temperature in 2 G LF. This small field was applied, instead of zero field, as a precautionary measure in order to suppress any effects from small residual stray fields $(50 \mathrm{mG})$ perpendicular to the initial muon polarization after the field zeroing procedure. However, $2 \mathrm{G}$ is not large enough to completely suppress effects from quasi-static nuclear dipolar fields.

Figure 3(a) shows spectra in $2 \mathrm{G} \mathrm{LF}$ at various temperatures. With decreasing temperature, the signal evolves from a simple Gaussian shape at high temperatures to a more complex form at low temperatures with at least two components. The Gaussian-shaped depolarization at high temperatures is interpreted to be due to randomly oriented proton nuclear magnetic moments in the thiol capping layer. The proton nuclear magnetic moment $\mu_{p}=2.793 \mu_{N}$ is 19 times larger than the gold nuclear magnetic moment, ${ }^{17}$ which plays no significant role in $2 \mathrm{G} \mathrm{LF}$. Electronic magnetic moments are assumed to fluctuate too fast to have a significant effect on the muon spin depolarization in the high-temperature regime. With decreasing temperature, a fast relaxing component develops, which is attributed to a slowing down of fluctuating electronic magnetic moments. As the fluctuating rate decreases with temperature, there is a larger spectral component at the muon Larmor frequency, and thus, more relaxation is observed. An additional possibility is that the moment size grows with decreasing temperature.

Figure 3(b) shows a temperature scan in a LF of $10 \mathrm{G}$ between $194 \mathrm{~K}$ and $4 \mathrm{~K}$. The signal can be fit to two components, an exponentially relaxing and a static part,

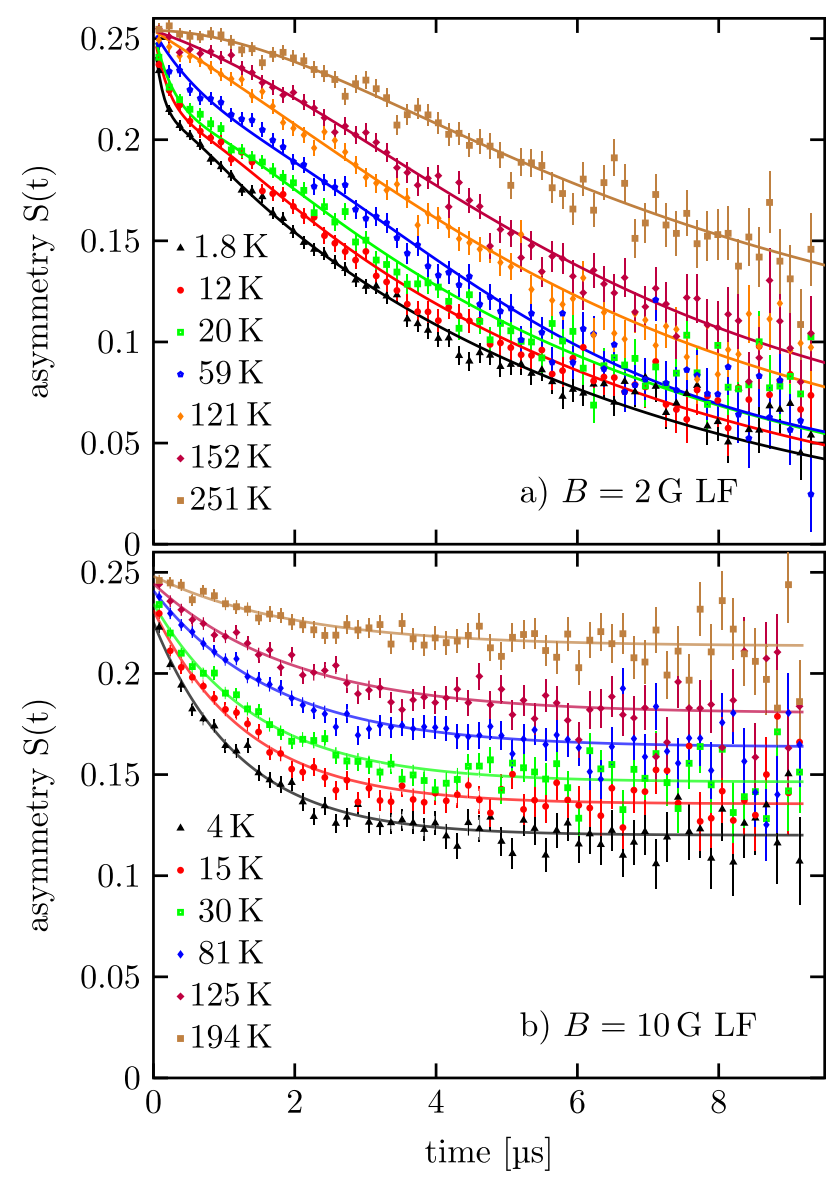

FIG. 3. (a) $2 \mathrm{G}$ LF $\mu \mathrm{SR}$ spectra: with decreasing temperature, the simple Gaussian-shaped damping, that is attributed to proton nuclear magnetic moments, is lost, and a fast relaxing front end develops. This is interpreted as a slowing down of fluctuating electronic moments. (b) $10 \mathrm{G} \mathrm{LF} \mu \mathrm{SR}$ spectra: nuclear contributions to the depolarization can be neglected in this field.

$$
S^{\mathrm{LF}}(t)=A_{\text {relax }} \exp \left(-\lambda^{\mathrm{LF}} t\right)+A_{\text {static }} .
$$

All dipolar fields from nuclear magnetic moments are negligible compared to the applied $10 \mathrm{G} \mathrm{LF}$, and thus, the observed relaxation is due solely to the electronic moments [Fig. 3(b)]. With decreasing temperature, the static, non-relaxing component gets smaller and the amplitude of the relaxing component increases. This is not consistent with a simple slowing down of fluctuating moments in a homogeneous system since in that case one would expect the amplitudes of the static and relaxing components to remain constant. The observed behaviour implies that the system is magnetically inhomogeneous in such a way that the fraction of muons experiencing depolarization on the muon time scale grows with decreasing temperature. This kind of behaviour may be expected in an inhomogeneous system with a broad range of correlation times which evolve as a function of temperature. A possible explanation is the existence of a crossover temperature $T_{X}$ such that there is no observed relaxation on the muon time scale above $T_{X}$ but significant relaxation below $T_{X}$. In a heterogeneous system, where $T_{X}$ depends on the location within the sample, i.e., in which particular GNP the muon stops, one expects the amplitudes of the relaxing and non-relaxing components to evolve with temperature as observed.

If the moments in GNPs were behaving collectively as superparamagnets, ${ }^{18} T_{X}$ could be associated with a 
superparamagnetic blocking temperature $T_{B}$, which controls the fluctuation rate for a given moment and temperature. In general, $T_{B}$ is expected to show a strong dependence on size and anisotropy, i.e., shape; ${ }^{19}$ a broad range of fluctuations as observed is therefore conceivable. However, at this point, we do not have conclusive evidence for superparamagnetism, let alone collective behaviour. For example, a very inhomogeneous paramagnet (without any giant spins) could exhibit the same behaviour as a function of temperature. Also, it seems unclear whether the term superparamagnetism, describing collective fluctuations in single-domain ferromagnetic particles with uniform magnetization, ${ }^{18}$ can accurately describe the behaviour of spatially inhomogeneous magnetic moments in GNPs. The use of $T_{B}$, and conclusions about magnetic anisotropy based on $T_{B}$, may therefore not be appropriate.

Spectra in LFs ranging from $2 \mathrm{G}$ to $2 \mathrm{kG}$ at $1.8 \mathrm{~K}$ are shown in Fig. 4. Again, the curves are fit to an exponential component plus a constant [Eq. (2)]. It is clear that the static component increases with the applied field: $10 \mathrm{G}$ is enough to recover $60 \%$ of the polarization; however, fields up to $2 \mathrm{kG}$ are required to completely quench the relaxation. These results are clear evidence that the observed relaxation is due to fluctuating electronic moments with a wide range of correlation times, which also explains the change in the ratio of static and dynamic fractions as a function of field.

From the present data, we cannot differentiate whether the moments are located at the surface or somewhere random within the GNP. However, it is worth noting that we recently showed conclusively that we can place muons onto the surface of uncapped GNPs in a silica matrix, ${ }^{20}$ thus using the muon as a uniquely sensitive probe for surface magnetism.

Summarizing, temperature-dependent magnetism that is highly disordered in space and time was observed in thiolcapped $2.2 \mathrm{~nm}$ gold nanoparticles. A highly inhomogeneous and dynamic internal field distribution of electronic origin is evident from the stretched exponential envelope of the TF signal and the multi-component LF signal. The response of the relaxation rate to an applied LF implies that the moments

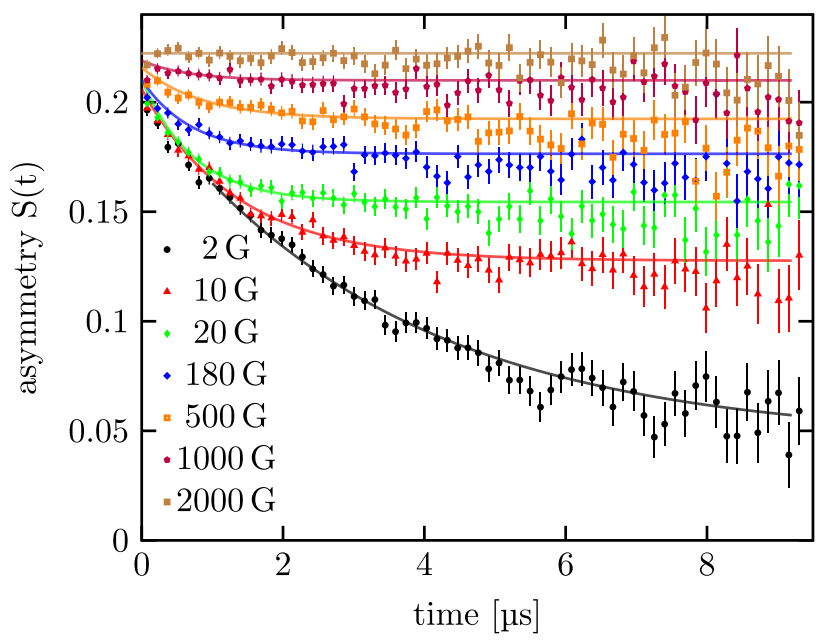

FIG. 4. $\mu$ SR spectra at $T=1.8 \mathrm{~K}$ in a LF scan ranging from $2 \mathrm{G}$ to $2 \mathrm{kG}$. The static tail increases with the applied field. A static distribution of magnetic moments that could explain the low field polarization would be completely quenched in fields of less than $100 \mathrm{G}$. Since this is not observed, the relaxation must originate from a rapidly fluctuating magnetic field of the electronic origin. responsible for the relaxation rapidly fluctuate with a broad distribution of correlation times. Also, there is a strong temperature-dependence attributed to a slowing down of the fluctuation rate. Finally, contrary to the findings of Goikolea, ${ }^{21}$ no coherent oscillations from the internal field could be observed.

It seems clear that the magnetic properties of a nanoparticle depend sensitively on the number of electrons or the density of states at the Fermi level. Since this depends on the number and the type of ligands, one may expect a large variability in the magnetic properties from one particle to the next. ${ }^{4,6,12}$ This appears to be consistent with the present experimental findings in which the magnetism is shown to be very inhomogeneous. Although there are reports of temperature dependent SQUID and XMCD measurements, ${ }^{5,7,11,22}$ Nealon et al. conclude in their extensive review that one common feature of the majority of experimental studies on GNPs is that the magnetism is at most weakly temperature dependent. ${ }^{4}$ However, the fluctuations in the magnetism we observe have a clear temperature dependence and slow down considerably at low temperature. This may be related to the fact that no other method is as sensitive to fluctuations in the range $10^{4}$ to $10^{12} \mathrm{~Hz}$ as $\mu \mathrm{SR}$.

Finally, we note that the results presented are an initial study using $\mu \mathrm{SR}$ to probe the magnetic properties of thiolcapped gold nanoparticles. Nevertheless, it has become clear that this method is well suited to this problem and can provide unique information on local magnetic properties that could help to disentangle different contributions of size, shape, and capping material and ultimately shed light on the precise nature and origin of magnetism in gold nanoparticles.

This research was performed at the TRIUMF Centre for Materials and Molecular Science. The authors thank B. Hitti, R. Abasalti, and D. Vyas for excellent technical support. We further thank M. Cortie at UTS for helpful discussions. We would also like to acknowledge NSERC (Canada) for financial support (Discovery Grants for R.F.K., W.A.M., and D.G.F).

${ }^{1}$ M. C. Daniel and D. Astruc, Chem. Rev. 104, 293 (2004).

${ }^{2}$ Y.-C. Yeh, B. Creran, and V. M. Rotello, Nanoscale 4, 1871 (2012).

${ }^{3}$ G. C. Bond and D. T. Thompson, Cat. Rev.- Sci. Eng. 41, 319 (1999).

${ }^{4}$ G. L. Nealon, B. Donnio, R. Greget, J.-P. Kappler, E. Terazzi, and J.-L. Gallani, Nanoscale 4, 5244 (2012).

${ }^{5}$ Y. Yamamoto, T. Miura, M. Suzuki, N. Kawamura, H. Miyagawa, T. Nakamura, K. Kobayashi, T. Teranishi, and H. Hori, Phys. Rev. Lett. 93, 116801 (2004).

${ }^{6}$ A. Ayuela, P. Crespo, M. A. Garca, A. Hernando, and P. M. Echenique, New J. Phys. 14, 013064 (2012).

${ }^{7}$ J. Bartolomé, F. Bartolomé, L. M. García, A. I. Figueroa, A. Repollés, M. J. Martínez-Pérez, F. Luis, C. Magén, S. Selenska-Pobell, F. Pobell, T. Reitz, R. Schönemann, T. Herrmannsdörfer, M. Merroun, A. Geissler, F. Wilhelm, and A. Rogalev, Phys. Rev. Lett. 109, 247203 (2012).

${ }^{8}$ M. Zhu, C. M. Aikens, M. P. Hendrich, R. Gupta, H. Qian, G. C. Schatz, and R. Jin, J. Am. Chem. Soc. 131, 2490 (2009), pMID: 19178295.

${ }^{9}$ J. S. Garitaonandia, M. Insausti, E. Goikolea, M. Suzuki, J. D. Cashion, N. Kawamura, H. Ohsawa, I. G. de Muro, K. Suzuki, F. Plazaola, and T. Rojo, Nano Lett. 8, 661 (2008).

${ }^{10}$ P. Crespo, R. Litrán, T. C. Rojas, M. Multigner, J. M. de la Fuente, J. C. Sánchez-López, M. A. García, A. Hernando, S. Penadés, and A. Fernández, Phys. Rev. Lett. 93, 087204 (2004).

${ }^{11}$ Y. Yamamoto and H. Hori, Rev. Adv. Mater. Sci. 12, 23 (2006); http:// www.ipme.ru/e-journals/RAMS/no_11206/yamamoto.pdf.

${ }^{12}$ A. Hernando, P. Crespo, M. A. Garca, M. Coey, A. Ayuela, and P. M. Echenique, Phys. Status Solidi B 248, 2352 (2011). 
${ }^{13}$ A. Yaouanc and P. D. de Rotier, Muon Spin Rotation, Relaxation, and Resonance: Applications to Condensed Matter (Oxford University Press, 2011).

${ }^{14}$ C. Kaiser, P. Gubbens, F. Mulder, P. D. de Rotier, A. Yaouanc, P. Paulus, L. de Jongh, G. Schmid, P. King, and A. Amato, Phys. B: Condens. Matter 326, 484 (2003).

${ }^{15}$ Y. Wu, Y. Li, P. Liu, S. Gardner, and B. S. Ong, Chem. Mater. 18, 4627 (2006).

${ }^{16}$ D. Mitchell, Micron 73, 36 (2015).

${ }^{17}$ NIST. The NIST Reference on Constants, Units, and Uncertainties (NIST, 2015).
${ }^{18}$ C. P. Bean and J. D. Livingston, J. Appl. Phys. 30, S120 (1959).

${ }^{19}$ G. C. Papaefthymiou, Nano Today 4, 438 (2009).

${ }^{20}$ M. H. Dehn, D. J. Arseneau, P. Böni, M. D. Bridges, T. Buck, D. L. Cortie, D. G. Fleming, J. A. Kelly, W. A. MacFarlane, M. J. MacLachlan, R. M. L. McFadden, G. D. Morris, P.-X. Wang, J. Xiao, V. M. Zamarion, and R. F. Kiefl, J. Chem. Phys. 145, 181102 (2016).

${ }^{21}$ E. Goikolea, J. Garitaonandia, M. Insausti, J. Lago, I. G. de Muro, J. Salado, F. Bermejo, and D. Schmool, J. Non-Cryst. Solids 354, 5210 (2008).

${ }^{22}$ P. Dutta, S. Pal, M. S. Seehra, M. Anand, and C. B. Roberts, Appl. Phys. Lett. 90, 213102 (2007). 